\title{
Study of a Light NMSSM CP-Odd Higgs Produced via Bottom-Quark Annihilation in the Di-Photon Channel at the LHC
}

\author{
M. M. Almarashi \\ Physics Department, Faculty of Science, Taibah University, P. O. Box 344, Medina, KSA
}

\begin{abstract}
We study the production of a low mass CP-odd Higgs through bottom-quark annihilation in the $\gamma \gamma$ final state at the LHC in the framework of the NMSSM. This production channel is significantly enhanced at large values of $\tan \beta$. We provide some results about the inclusive cross section of this production mode, which may help for extracting the $a_{1}$ signal at the LHC.
\end{abstract}

Keywords: supersymmetric models, CP-odd Higgs bosons, NMSSM, LHC

DOI: 10.31526/ACP.BSM-2021.17

\section{INTRODUCTION}

The discovery of the a Standard-Model-like Higgs boson with a mass of approximately $125 \mathrm{GeV}$ at the CERN Large Hadron Collider (LHC) $[1,2]$ opens up a new window to explore an extended Higgs sector, with additional (pseudo)scalar Higgs bosons, predicted by several theories such as the Next-to-Minimal Supersymmetric Standard Model (NMSSM) [3, 4, 5, 6, 7, 8]. The NMSSM is quite an attractive model. First, it provides a natural solution to the mu-problem of the MSSM by adding a chiral singlet superfield to the MSSM [3]. Second, it can relieve the Little Hierarchy Problem and Fine-tuning [9, 10]. Third, the phenomenology of the NMSSM is really richer than that of the MSSM, particularly in the the Higgs sector. Hence, the NMSSM deserves more attention.

After electroweak symmetry breaking (EWSB), the NMSSM Higgs sector consists of seven Higgs bosons, three of which are $\mathrm{CP}$-even, two are $\mathrm{CP}$-odd and two charged states. The tree-level Higgs sector of this model can be described by six parameters: the Yukawa couplings $\lambda$ and $\kappa$, the associated trilinear soft-breaking parameters $A_{\lambda}$ and $A_{\kappa}$, the parameters $\mu_{\text {eff }}=\lambda s$ with $s$ being the vacuum expectation value of the singlet field and $\tan \beta \equiv v_{u} / v_{d}$ with $v_{u}$ and $v_{d}$ being the vacuum expectation values of the Higgs doublets.

Following the discovery of the Higgs boson in 2012 at the LHC, looking for non SM Higgs bosons, if exist, is crucial in probing physics beyond the SM. Of particular interest is a light CP-odd Higgs boson. A recent detailed study of search for a light CP-odd Higgs boson via its production in bottom-gluon fusion in the framework of the NMSSM at the LHC can be found in [11].

In this paper we study the potential discovery of a light CP-odd Higgs particle at the LHC through bottom quark fusion $b \bar{b} \rightarrow a_{1}$, followed by the decay $a_{1} \rightarrow \gamma \gamma$, Figure 1, in the NMSSM framework. The initial bottom quarks reside in the proton sea, and the bottom-quark sea is generated from the splitting of gluons into nearly-collinear bottom-antibottom pairs. The $a_{1}$ decay into two photons is mediated by loops of fermions. If the $a_{1}$ is a singlet-like then the chargino contribution is the dominant one. This work is complementary to the one carried out in Ref. [12], in which we explored the production channel $b \bar{b} \rightarrow a_{1} \rightarrow \tau^{+} \tau^{-}$of such a low-mass $a_{1}$ state. In the next section we give a brief review of the NMSSM, focusing on the light CP-odd Higgs particle inside this model. The results and discussions are presented in section 3. Finally, conclusions are given in section 4.

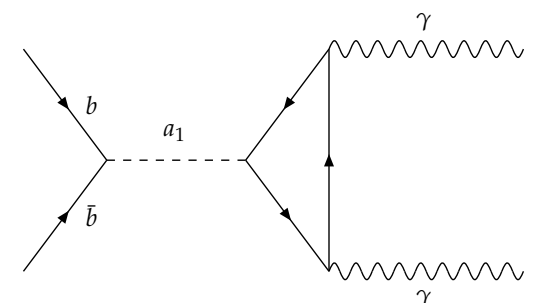

FIGURE 1: Representative Feynman diagram for the lightest CP-odd Higgs production with subsequent decay to a pair of photons $b \bar{b} \rightarrow a_{1} \rightarrow \gamma \gamma$.

\section{LIGHT CP-ODD HIGGS BOSON OF THE NMSSM}

The NMSSM superpotential depends on the usual two Higgs doublets superfields of the MSSM $\hat{H}_{u}$ and $\hat{H}_{d}$ and the additional singlet one $\hat{S}$. It is given by

$$
W_{N M S S M}=W_{M S S M}+\lambda \hat{S} \hat{H}_{u} \hat{H}_{d}+\frac{1}{3} \kappa \hat{S}^{3},
$$


where $W_{M S S M}$ is known MSSM superpotential. Correspondingly, the soft supersymmetric breaking terms for the NMSSM Higgs sector read

$$
V_{\mathrm{NMSSM}}=m_{H_{u}}^{2}\left|H_{u}\right|^{2}+m_{H_{d}}^{2}\left|H_{d}\right|^{2}+m_{S}^{2}|S|^{2}+\left(\lambda A_{\lambda} S H_{u} H_{d}+\frac{1}{3} \kappa A_{\kappa} S^{3}+\text { h.c. }\right) .
$$

The lightest CP-odd Higgs boson of the NMSSM results from the mixing between the CP-odd Higgs state of the MSSM $a_{M S S M}$ and the CP-odd singlet state of the NMSSM:

$$
a_{1}=a_{\mathrm{MSSM}} \cos \theta_{A}+a_{\mathrm{S}} \sin \theta_{A}
$$

where $\theta_{A}$ is the mixing angle.

A light $a_{1}$ is naturally accommodated in the NMSSM parameter space, which is of great interest. The tree-level mass-squared of the $a_{1}$, to a good approximation, are given by the following expressions:

$$
m_{a_{1}}^{2}=-\frac{3 \kappa \mu_{\mathrm{eff}} A_{\kappa}}{\lambda}+v^{2} \sin 2 \beta\left(2 \lambda \kappa+\frac{A_{\lambda} \lambda^{2}}{\sqrt{2} \mu_{e f f}}\right),
$$

where $v \equiv \sqrt{v_{u}^{2}+v_{d}^{2}}$, with $v_{u}$ and $v_{d}$ are the vacuum expectation values for up-type and down type Higgs doublets as mentioned above. One can see from Eq. 4 that all the tree-level Higg sector parameters affects on $m_{a_{1}}$.

We try to find the region of the NMSSM parameter space that offers a light $a_{1}$. We randomly scan the parameter space by using the NMSSMTools_5.5.2 package $[13,14,15,16]$ with the goal of finding such the light $a_{1}$. Details of our parameter space scan including theoretical and experimental constraints can be found in Ref. [12].

\section{RESULTS AND DISCUSSIONS}

The $\gamma \gamma$ signal may be the most ideal decay mode to search for a light $a_{1}$ at the LHC as a hadron collider suffering from large hadron backgrounds. The light $a_{1}$ dominantly decays to $b \bar{b}$ with branching ratio close to $90 \%$ for most points of the NMSSM parameter space but this channel suffers from large QCD backgrounds. The branching ratio of the light $a_{1}$ decay into di-tau decay channel is $\lesssim 10 \%$ in most of the parameter space when the $b \bar{b}$ decay channel is kinematically open. The di-tau decay channel was studied for the $a_{1}$ production via the bottom-quark fusion, see [12]. In this work we study the production process $b \bar{b} \rightarrow a_{1}$ followed by $a_{1} \rightarrow \gamma \gamma$. Presence of the two photons in the final state is very encouraging since it provides a very clean signature for probing $a_{1}$ properties such as its mass.

As a first step, we show in Fig. 2 the correlations between the mass of the lightest CP-odd Higgs boson, $m_{a_{1}}$, and the lightest two CP-even Higgs masses, $m_{h_{1}}$ and $m_{h_{2}}$, (top two panels) and between the former one and the branching fraction $\operatorname{BR}\left(a_{1} \rightarrow \gamma \gamma\right)$. It is clear from the top-panel that in our parameter space the $h_{1}$ is mostly the SM-like Higgs with masses between 122 and 128 $\mathrm{GeV}$ though one can not generalize this to the entire parameter space where the $h_{1}$ can also be a singlet-like with masses less than that of the SM Higgs boson. It is also obvious from the middle-panel of the figure that the $h_{2}$ can play the role of the SM-like Higgs in a small region of the parameter space. Moreover, it is also noticeable that the smaller $m_{a_{1}}$ the smaller $m_{h_{2}}$ Furthermore, it is remarkable that there is a certain region of the NMSSM parameter space in which the $B R\left(a_{1} \rightarrow \gamma \gamma\right)$ is dominant, reaching up to $100 \%$, see the bottom-panel of the figure. The reason behind this is the singlet nature of the $a_{1}$ leading to suppressions of the $a_{1}$ decays to fermion-antifermion at the tree level like $a_{1} \rightarrow b \bar{b}$ and $a_{1} \rightarrow \tau^{+} \tau^{-}$in which case the $a_{1} \chi_{1}^{+} \chi_{1}^{-}$coupling generating through the $\lambda H_{1} H_{2} S$ Lagrangian term is enhanced, inducing the enhancement of the $\gamma \gamma$ decay channel. It is further noticed that the $\operatorname{BR}\left(a_{1} \rightarrow \gamma \gamma\right)$ ranges from $10^{-3}$ to $10^{-6}$ for most points in our parameter space.

In order to study the discovery potential of the $a_{1}$ at $14 \mathrm{TeV}$ center-of-mass energy at the LHC, we evaluate the inclusive production rates of the $a_{1}$ by using CalcHEP [17] for the points surviving the constraints in the scan. We focus on the production channel $b \bar{b} \rightarrow a_{1}$. This channel is significantly enhanced at large values of $\tan \beta$. Figure 3 presents the production rates $\sigma(b \bar{b} \rightarrow$ $\left.a_{1}\right) \mathrm{BR}\left(a_{1} \rightarrow \gamma \gamma\right)$ as functions of $a_{1}$ mass (top), $\operatorname{BR}\left(a_{1} \rightarrow \gamma \gamma\right)$ (middle) and $\tan \beta$ (bottom). The top-panel of the figure shows that the production rates decreases by increasing the $a_{1}$ mass, as expected. This production rates reach up to $10^{8}$ fb for large values of $\tan \beta$, see the bottom-panel of the figure. The middle-panel of the figure shows that the $\operatorname{BR}\left(a_{1} \rightarrow \gamma \gamma\right)$ can be enhanced in some region of the NMSSM parameter space, reaching up to $100 \%$. This enhancement of the $\operatorname{BR}\left(a_{1} \rightarrow \gamma \gamma\right)$ is a characteristic feature of the NMSSM compared with the other SUSY models. Unfortunately, this region does not correspond to the one that maximizes the inclusive production rates $\sigma\left(b \bar{b} \rightarrow a_{1}\right) \operatorname{BR}\left(a_{1} \rightarrow \gamma \gamma\right)$. This is because the production channel $b \bar{b} \rightarrow a_{1}$ is suppressed in contrast to the decay channel $a_{1} \rightarrow \gamma \gamma$ which is enhanced. In fact, the maximum cross sections occurs when the $\operatorname{BR}\left(a_{1} \rightarrow \gamma \gamma\right)$ ranges from $10^{-4}$ to $5 \times 10^{-5}$. In summary, we conclude that although the details of signal-to-background analysis is required to make the final conclusion about the discovery potential of $a_{1}$ via the process $b \bar{b} \rightarrow a_{1} \rightarrow \gamma \gamma$ at the LHC, the total production rates for the production channel $b \bar{b} \rightarrow a_{1}$ followed by the decay $a_{1} \rightarrow \gamma \gamma$ are quite large, and may help extracting the $a_{1}$ signals at least at high-Luminosities of the LHC. 

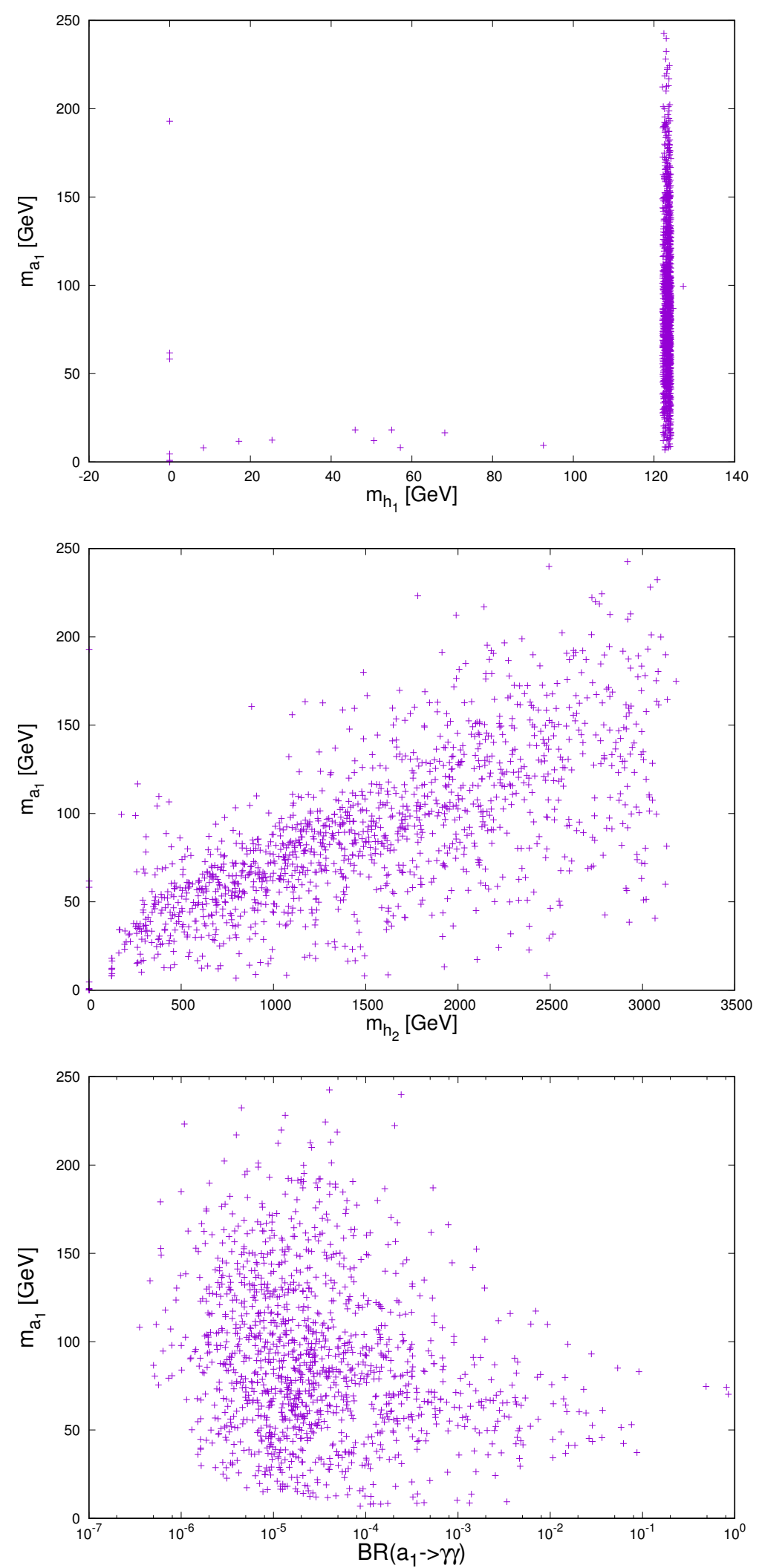

FIGURE 2: The correlations between the lightest CP-odd Higgs mass, $m_{a_{1}}$ and the lightest two CP-even Higgs masses, $m_{h_{1}}$ and $m_{h_{2}}$ and between the former one and and the di-photon decay rate. 

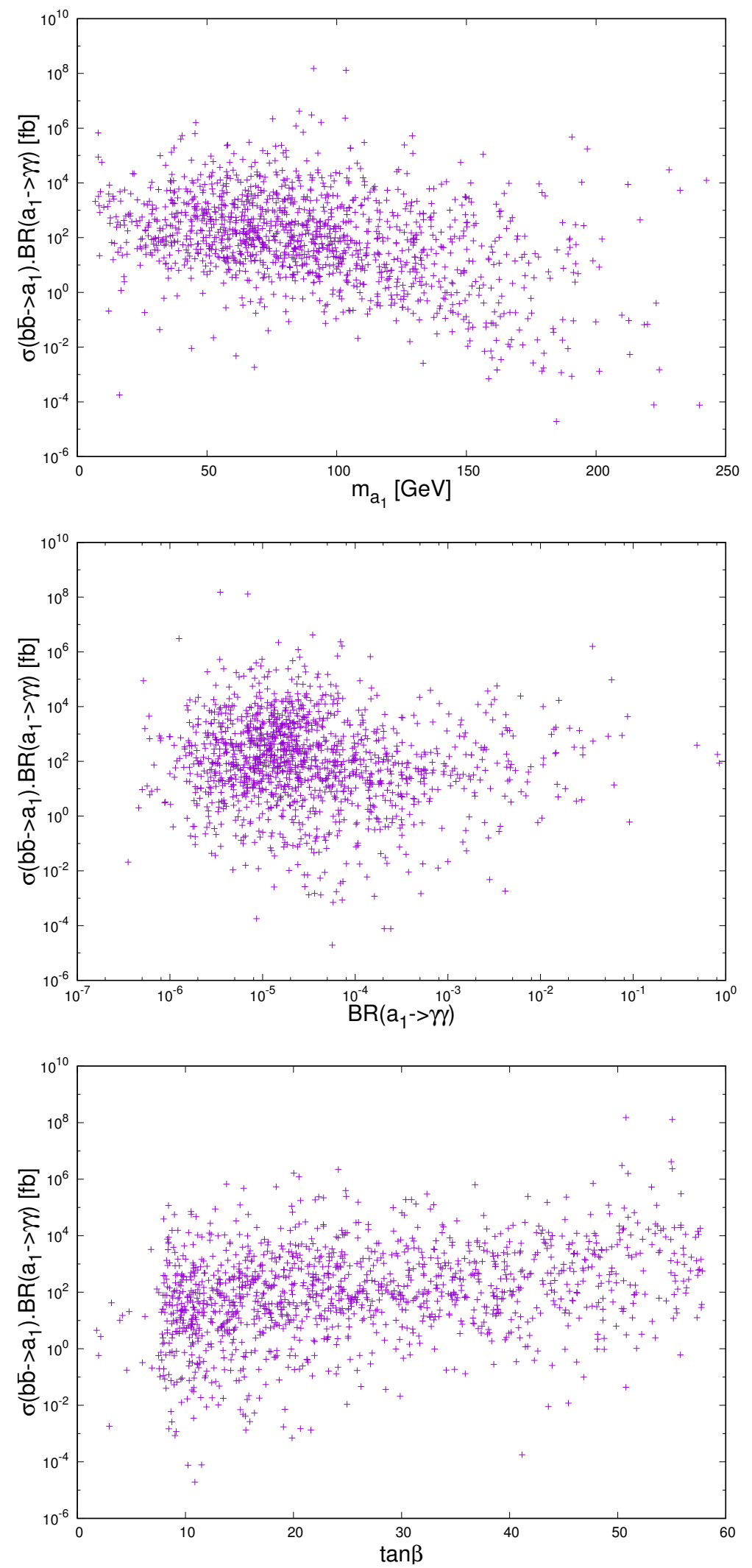

FIGURE 3: The production rates of the lightest CP-odd Higgs boson $a_{1}$ produced in bottom-gluon fusion $\sigma\left(g g \rightarrow a_{1}\right) \mathrm{BR}\left(a_{1} \rightarrow \gamma \gamma\right)$ versus $a_{1}$ mass (top), $\mathrm{BR}\left(a_{1} \rightarrow \gamma \gamma\right)$ (middle) and $\tan \beta$ (bottom) at $14 \mathrm{TeV}$ LHC for the points surviving the constraints in the random scan. 


\section{CONCLUSIONS}

In this work, we have studied the production of a light CP-odd Higgs boson, $a_{1}$, of the Next-to-Minimal Supersymmetric Standard Model (NMSSM), which contains a singlet superfields in addition to the two doublet ones of the MSSM, at the LHC. We have estimated the inclusive production rates for the $a_{1}$ produced in bottom-quark fusion through di-photon decay channel. We have found that these production rates are quite large, and could help extracting the di-photon $a_{1}$ signal, at least at the High-Luminosity LHC. The discovery of such a light $a_{1}$ with mass less than $Z$ mass is not only an evidence of supersymmetric models but it can also distinguish the NMSSM from the MSSM. The $a_{1}$ production through bottom-quark fusion in the di-photon final state is enhanced at large values of $\tan \beta$, and can be exploited to measure both the $a_{1}$-to-bottom-antibottom $a_{1} b \bar{b}$ coupling and the effective $a_{1}$-todiphoton coupling at the LHC.

\section{ACKNOWLEDGEMENTS}

This work is supported by Taibah University.

\section{References}

[1] S. Chatrchyan et al. [CMS Collaboration], Phys. Lett. B 716, 30 (2012).

[2] G. Aad et al. [ATLAS Collaboration], Phys. Lett. B 716, 1 (2012).

[3] J. R. Ellis, J. F. Gunion, H. E. Haber, L. Roszkowski and F. Zwirner, Phys. Rev. D 39, 844 (1989).

[4] M. Drees, Int. J. Mod. Phys. A 4, 3635 (1989).

[5] U. Ellwanger, M. Rausch de Traubenberg and C. A. Savoy, Phys. Lett. B 315, 331 (1993).

[6] S. F. King and P. L. White, Phys. Rev. D 52, 4183 (1995).

[7] F. Franke and H. Fraas, Int. J. Mod. Phys. A 12, 479 (1997).

[8] U. Ellwanger, M. Rausch de Traubenberg and C. A. Savoy, Nucl. Phys. B 492, 21 (1997).

[9] M. Bastero-Gil, C. Hugonie, S. F. King, D. P. Roy and S. Vempati, Phys. Lett. B 489359 (2000).

[10] R. Dermisek and J. F. Gunion, Phys. Rev. Lett. 95041801 (2005).

[11] M. M. Almarashi, Int. J. Mod. Phys. A 35, no.25, 2050151 (2020).

[12] M. M. Almarashi, Results Phys 23 (2021) 104022.

[13] U. Ellwanger, J.F. Gunion and C. Hugonie, JHEP 0502, 066 (2005).

[14] U. Ellwanger and C. Hugonie, Comput. Phys. Commun. 175, 290 (2006).

[15] See https://www.lupm.univ-montp2.fr/users/nmssm/.

[16] F. Domingo, JHEP 06, 052 (2015).

[17] A. Belyaev, N. D. Christensen and A. Pukhov, Comput. Phys. Commun. 184, 1729 (2013). 